\title{
Treinamento inicial em microcirurgia
}

\section{Initial training in microsurgery}

Trabalho realizado no Serviço de Cirurgia Plástica e Microcirurgia Reconstrutiva do Hospital Universitário Walter Cantídio, Fortaleza, CE, Brasil.

Artigo submetido no SGP (Sistema de Gestão de Publicações) da RBCP.

Artigo submetido: 5/2/2010 Artigo aceito: $26 / 11 / 2010$

\begin{abstract}
RESUMO
A técnica microcirúrgica que até a década de 80 do século passado se constituiu um procedimento de exceção, somente utilizada em casos extremos, devido aos avanços facilmente observados em diversas publicações atuais, tornou-se em muitos procedimentos a primeira opção cirúrgica, fazendo com quase que, de maneira obrigatória, todos os médicos que exerçam a especialidade de Cirurgia Plástica tenham que realizar treinamento na citada técnica operatória. O presente artigo se propõe a apresentar um modelo de fácil execução como treinamento básico em microcirurgia.
\end{abstract}

Descritores: Cirurgia plástica. Microcirurgia. Educação médica.

\section{ABSTRACT}

The microsurgical technique witch until the 1980's was an exclusion procedure, only chosen in extreme cases, due to easily observed advances seen in innumerous current publications, has become in various procedures the first surgical option, practically compelling all doctors who practice in the field of Plastic Surgery to perform training in the referred technique. The current article presents an easy executed model of microsurgery basic training.

Keywords: Surgery, plastic. Microsurgery. Education, medical.

\section{INTRODUÇÃO}

A microcirurgia pode ser definida como um conjunto de procedimentos cirúrgicos realizados com o auxílio de um meio óptico de magnificação ${ }^{1-3}$. É uma técnica que pode ser aplicada a inúmeras áreas da cirurgia e cuja indicação abrange diversas especialidades, da Oftalmologia à Cirurgia Plástica ${ }^{4-7}$.

A introdução do uso de magnificação na prática cirúrgica em 1921, por Nylen, otorrinolaringologista sueco, permitiu a realização de procedimentos em estruturas cada vez menores ${ }^{1,8}$. A microcirurgia nasce na década de 60 do século passado, com as demonstrações de Jacobson e Suarez de anastomoses pérvias em vasos com diâmetros de 1,6 a 3,2 $\mathrm{mm}^{1,8}$, utilizando os princípios de suturas vasculares definidos por Carre1 ${ }^{9-11}$, em 1902. Em 1965, Buncke introduz a microcirurgia na Cirurgia Plástica, com o relato de um reimplante de orelha de coelho, realizando a anastomose em vasos de aproximadamente $1 \mathrm{~mm}$, tendo utilizado instrumentos adaptados daqueles utilizados por relojoeiros ${ }^{1,8,11}$. No Brasil, o pioneiro na utilização da microcirurgia foi Jorge Fonseca Ely, em $1968^{12}$. A introdução da microcirurgia vascular experimental ocorreu em 1971, no Laboratório de Microcirurgia na Faculdade de Medicina da Universidade de São Paulo ${ }^{8}$, o primeiro da América do Sul. O primeiro reimplante microcirúrgico em humanos com sucesso foi um reimplante de mão realizado por Ferreira et al., em 1972 2,8 .

O domínio da técnica microcirúrgica é um processo longo e difícil, que requer grande habilidade manual, paciência, dedicação e treinamento contínuo ${ }^{5,7,13}$. Existem vários modelos de treinamento que envolvem diferentes materiais e animais. A prática em materiais inertes, como látex, pode ser um importante passo inicial. O treinamento em animais, como ratos, camundongos, coelhos, cães e porcos, é essencial para o desenvolvimento de habilidades cirúrgicas ${ }^{13,14}$.

1. Médica Residente do Serviço de Cirurgia Plástica e Microcirurgia Reconstrutiva do Hospital Universitário Walter Cantídio da Universidade Federal do Ceará, Fortaleza, CE, Brasil; Membro Aspirante da Sociedade Brasileira de Cirurgia Plástica (SBCP).

2. Regente do Serviço de Cirurgia Plástica e Microcirurgia Reconstrutiva do Hospital Universitário Walter Cantídio da Universidade Federal do Ceará, São Lucas - Hospital de Cirurgia e Anestesia, Fortaleza, CE, Brasil; Membro Titular da SBCP.

3. Acadêmico de Medicina da Universidade Federal do Ceará, Fortaleza, CE, Brasil; Membro da Liga de Cirurgia Plástica e Microcirurgia Reconstrutiva Dr. Germano Riquet.

4. Chefe de Clínica do Serviço de Cirurgia Plástica e Microcirurgia Reconstrutiva do Hospital Universitário Walter Cantídio da Universidade Federal do Ceará, Fortaleza, CE, Brasil; Membro Titular da SBCP. 
Os avanços em pesquisa e ensino na área de microcirurgia permitiram a extensa aplicação desta técnica em diversas reconstruções ${ }^{15-17}$. A criação e a manutenção de serviços especializados são indispensáveis para a continuidade do treinamento e melhora gradativa dos resultados ${ }^{15,16}$.

O objetivo deste trabalho é apresentar uma proposta simples para treinamento inicial em microcirurgia.

\section{MÉTODO}

O Serviço de Cirurgia Plástica e Microcirurgia do Hospital Universitário Walter Cantídio da Universidade Federal do Ceará (UFC) desenvolveu, em 2009, uma proposta de treinamento inicial em microcirurgia.

Consistem em três etapas, sendo 60 horas destinadas às duas etapas básicas iniciais (Quadros 1 e 2).

A primeira etapa do treinamento é destinada à familiarização com o microscópio e os principais equipamentos e instrumentos utilizados na técnica microcirúrgica (Figura 1A). A segunda etapa concentra-se no treinamento de suturas em materiais inertes. A terceira etapa objetiva sedimentar os conhecimentos e habilidades adquiridos nas etapas anteriores, por meio do treinamento da técnica cirúrgica em modelos com segmentos de animais, com a realização de suturas vasculares. Treinamento com animais vivos, confecção e transferência de retalhos e o transplante de órgãos não foram incluídos nesta proposta de treinamento inicial.

A primeira etapa do treinamento é estruturada para que o cirurgião aprendiz conheça o instrumento fundamental da microcirurgia: o microscópio. Esta etapa repete-se durante todo o treinamento, no qual se deve aprender a manipular adequadamente o microscópio, com todos os ajustes necessários para um treinamento apropriado e, futuramente, para uma boa visualização do campo operatório.

Quadro 1 - Objetivos de cada etapa do treinamento.

\begin{tabular}{|c|c|}
\hline Etapa & Objetivos \\
\hline 1 & $\begin{array}{c}\text { Familiarização com o microscópio e com os } \\
\text { instrumentos }\end{array}$ \\
\hline $2 a$ & $\begin{array}{l}\text { Treinamento de suturas em látex - suturas } \\
\text { simples }\end{array}$ \\
\hline $2 b$ & $\begin{array}{l}\text { Treinamento de suturas em látex - confecção de } \\
\text { tubos para simulação de microanastomoses }\end{array}$ \\
\hline 3 & $\begin{array}{c}\text { Realização de suturas vasculares em peças } \\
\text { anatômicas }\end{array}$ \\
\hline
\end{tabular}

Quadro 2 - Instrumentos necessários às etapas iniciais do treinamento.

\section{Materiais necessários à primeira e segunda etapas}

1. Microscópio binocular cirúrgico com objetiva de $150 \mathrm{~mm}$ a $200 \mathrm{~mm}$

2. Suporte para o microscópio, com altura de 75 a $95 \mathrm{~cm} \mathrm{e}$ largura de 60 a $70 \mathrm{~cm}$

3. Assento sem encosto e sem braço

4. Material microcirúrgico

a. Pinça reta para suturas

b. Pinça curva

c. Pinça angulada para auxílio nas anastomoses término laterais

d. Porta-agulha

e. Tesoura reta para corte de fio

f. Tesoura curva para dissecção

g. Clamps vasculares (simples ou duplo com barra de aproximação)

h. Fios de Nylon 8-0, 9-0, 10-0 e 11-0.

5. Material macrocirúrgico
a. Cabo de bisturi $\mathrm{n}^{\circ} 3$
b. Lâminas de bisturi $\mathrm{n}^{\circ} 15$
c. Tesoura
d. Pinça dente de rato

6. Luvas de látex

7. Lupas de aumento

8. Palhetas de madeira

9. Material fotográfico

A segunda etapa do treinamento planeja permitir o aprendizado de suturas microcirúrgicas. Durante esta etapa, o cirurgião necessita habituar-se à visão bidimensional do microscópio, estimulando o reconhecimento de fatores do campo operatório que possam auxiliar na noção de profundidade perdida, aprender a correta forma de manipular os instrumentos microcirúrgicos e aprender a realizar sutura microcirúrgica.

Esta etapa é dividida em duas outras. A etapa $2 a$ é destinada ao aprendizado de suturas simples a serem realizadas em segmentos de luvas de látex (Figura 1B). A etapa $2 \mathrm{~b}$ foi destinada à simulação de realização de anastomoses em tubos de látex. Nesta, o cirurgião teve a oportunidade de realizar suturas em níveis crescentes de dificuldade, uma vez que a confecção do tubo e posterior simulação da anastomose fazem parte do procedimento. 

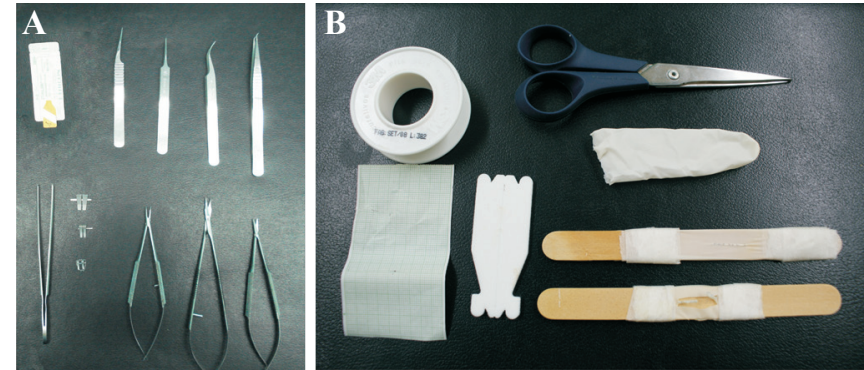

Figura 1 - A: Instrumental microcirúrgico; B: Outros materiais utilizados no treinamento.

A correta realização desta etapa deve seguir os seguintes passos.

Etapa 2a

a) Monte um segmento de luva de látex na palheta de madeira. Procure não manter o segmento de luva muito tensionado, pois isso pode prejudicar ou impossibilitar a aproximação de bordas ao tentar realizar a sutura nos passos seguintes;

b) Fixe o conjunto à mesa para evitar movimentos;

c) Comece o treinamento com a posição de escrever. Segure as pinças como se fossem canetas. Mantenha os ombros relaxados, com cotovelo, punho e borda ulnar do antebraço e da mão, apoiados na superfície, deixando somente as pontas dos dedos livres. Delicadamente, faça exercícios de fechar as pinças em ambas as mãos, com controle fino do movimento. O polegar e indicador fazem o movimento, enquanto dedo médio apóia a pinça;

d) Manipule a agulha com as pinças. Utilize a pinça reta na mão esquerda e a curva na mão direita;

e) Utilize agulhas microvasculares e nylon 8-0, 9-0, 10-0 e 11-0, fio com tamanho no máximo $10 \mathrm{~cm}$;

f) Estabilize a agulha mantendo um ângulo de 90 graus com a ponta da pinça;

g) Utilizando o conjunto palheta de madeira, segmento de luva de látex, fixado à bancada, realize uma incisão linear utilizando uma pinça, reta ou curva, e uma tesoura. Realize pontos separados com três nós, cada um. Fios de diferentes diâmetros devem ser utilizados, devendo iniciar com fios de diâmetro maior, como o $8-0$, pois apresentam dificuldade crescente na curva de aprendizado (Figura 2A);

h) Modificar a posição da palheta e repetir o procedimento. Posicionar a palheta em diferentes posições para evitar vícios de posição.

\section{Etapa 2b}

a) Monte um segmento de luva de látex na palheta de madeira;

b) Fixe o conjunto à mesa para evitar movimentos;

c) Realize duas incisões longitudinais paralelas no segmento de látex. A distância entre as duas incisões determinará o comprimento da circunferência do tubo e, portanto, o diâmetro;

d) Realize quatro incisões transversais, perpendiculares aos limites das incisões longitudinais, de modo que o segmento de látex incisado fique preso ao conjunto apenas pela porção central (Figura 2B);

e) Realize suturas simples para unir as duas bordas maiores do retângulo criado no item d (Figura 2C), resultando em um cilindro;

f) $\mathrm{O}$ diâmetro do cilindro pode ser calculado pela distância entre as bordas. Assim, para criar um cilindro de $1 \mathrm{~mm}$ de diâmetro, a distância entre as bordas deve ser de $3,14 \mathrm{~cm}$, uma vez que essa distância corresponde ao comprimento da secção transversal do cilindro criado e, portanto, obedece à fórmula matemática $\pi \mathrm{d}(\mathrm{d}=$ diâmetro da circunferência; $\pi=$ constante matemática de valor 3,14159265);

g) Seccione transversalmente o tubo formado no item f;

h) Posicione os clamps em cada segmento formado (Figura 2D);

i) Realize suturas simples para simular a anastomose na
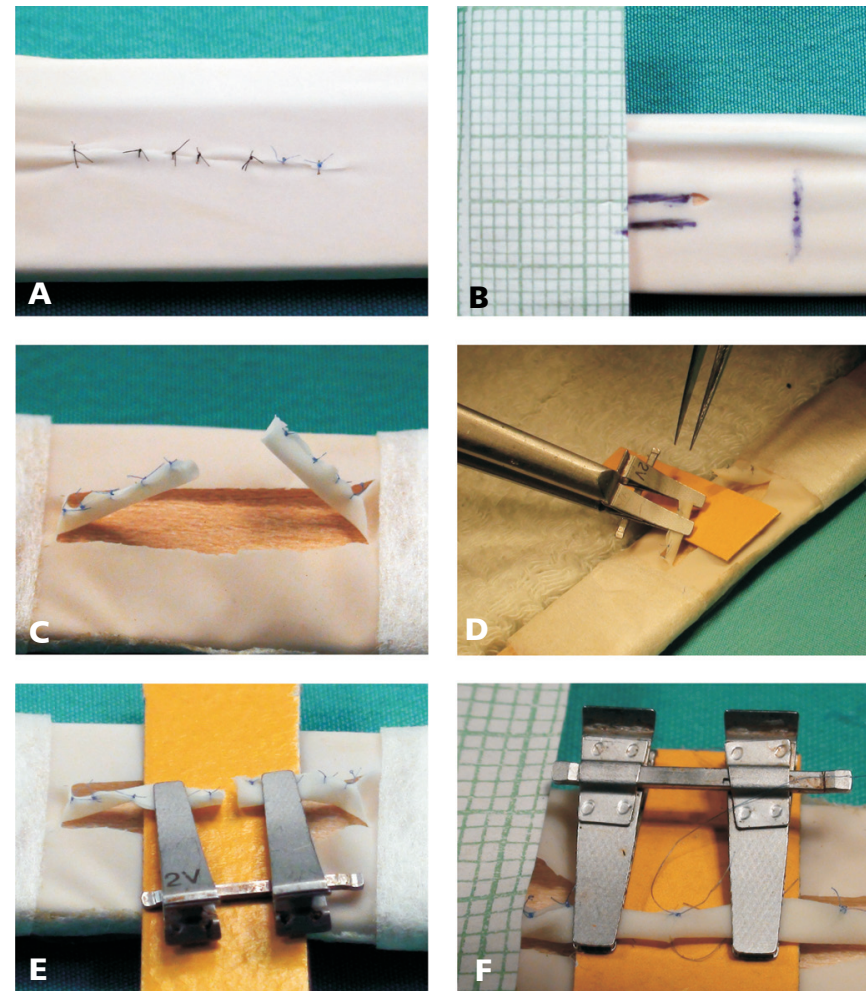

Figura 2 - Representação da etapa 2 do protocolo. A: Suturas simples realizadas após a incisão longitudinal no látex; B: Incisões longitudinais e transversais para a confecção do tubo de látex; C: Tubo de látex confeccionado após a realização de suturas simples na borda maior do retângulo; D: Posicionamento dos clamps no tubo de látex; E: Aproximação dos cotos para a anastomose do tubo; F: Anastomose dos cotos do tubo de látex. 
seguinte sequência: (1) Realize suturas localizadas a $0^{\circ}, 120^{\circ}$ e $240^{\circ}$; (2) Realize as demais suturas inicialmente na parede posterior do tubo de látex; (3) Realize uma sutura a $300^{\circ}$, seguida de uma a $180^{\circ}$ e outra a $60^{\circ}$ (Figura $2 \mathrm{E}$ e $2 \mathrm{~F}$ );

A terceira etapa do treinamento introduz ao cirurgião a capacidade de realizar suturas vasculares. Esta deve ser realizada em três etapas, das quais apenas a primeira será abordada neste protocolo. A etapa 3 consta da realização de suturas em vasos em peças anatômicas de animais (Figura $3 \mathrm{~A}$ ), visando estabelecer as bases para a aplicação do treinamento em humanos.

A correta realização desta etapa deve seguir os seguintes passos.

Etapa 3

a) Selecione vasos de diâmetros de $0,5 \mathrm{~mm}$ a $1,5 \mathrm{~mm}$, oriundos de segmentos de ratos, porcos ou aves, de modo a facilitar o treinamento (Figura 3B e 3C);

b) Posicione o clamp vascular de acordo com as Figuras $3 \mathrm{D}$ e $3 \mathrm{E}$;

c) Realiza a incisão do vaso com tesoura curva de microcirurgia (Figura 3F);

d) Prossiga com a ressecção da camada adventícia (Figura 4A);
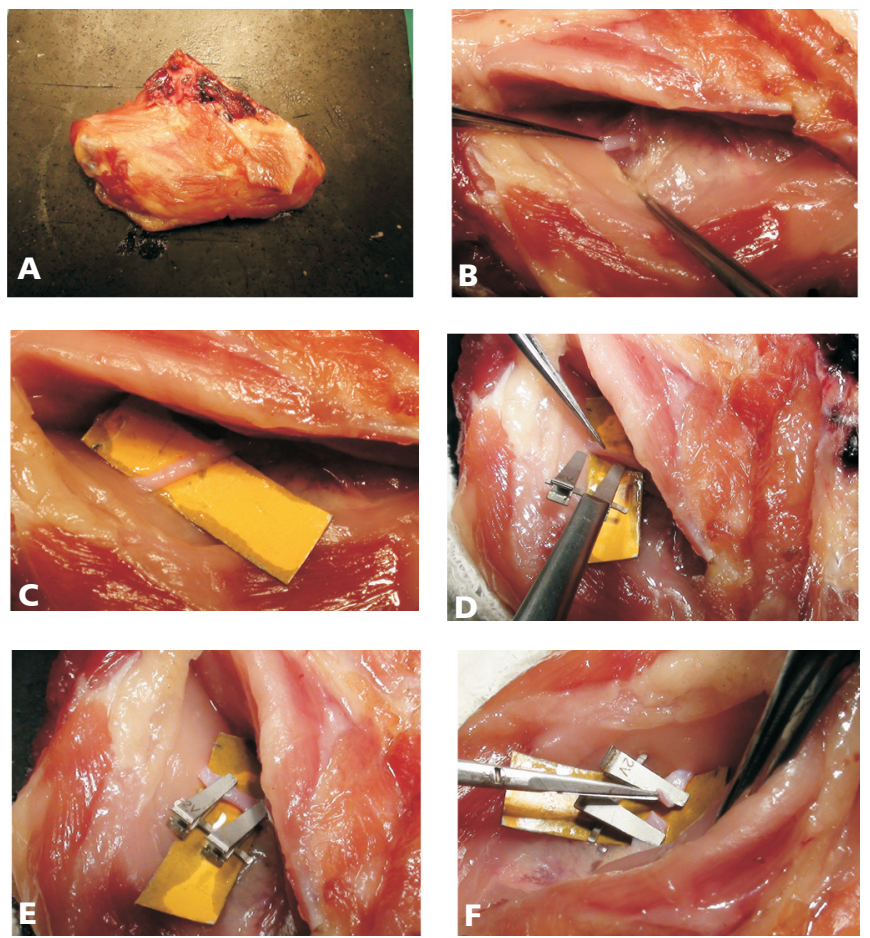

Figura 3 - Representação da etapa 3 a do protocolo. A: Peça anatômica fresca de frango; B: Localização e dissecção do vaso; $\boldsymbol{C}$ : Isolamento do vaso; $\boldsymbol{D}$ e E: Posicionamento dos clamps; $\boldsymbol{F}$ : Incisão no vaso para a realização da anastomose.
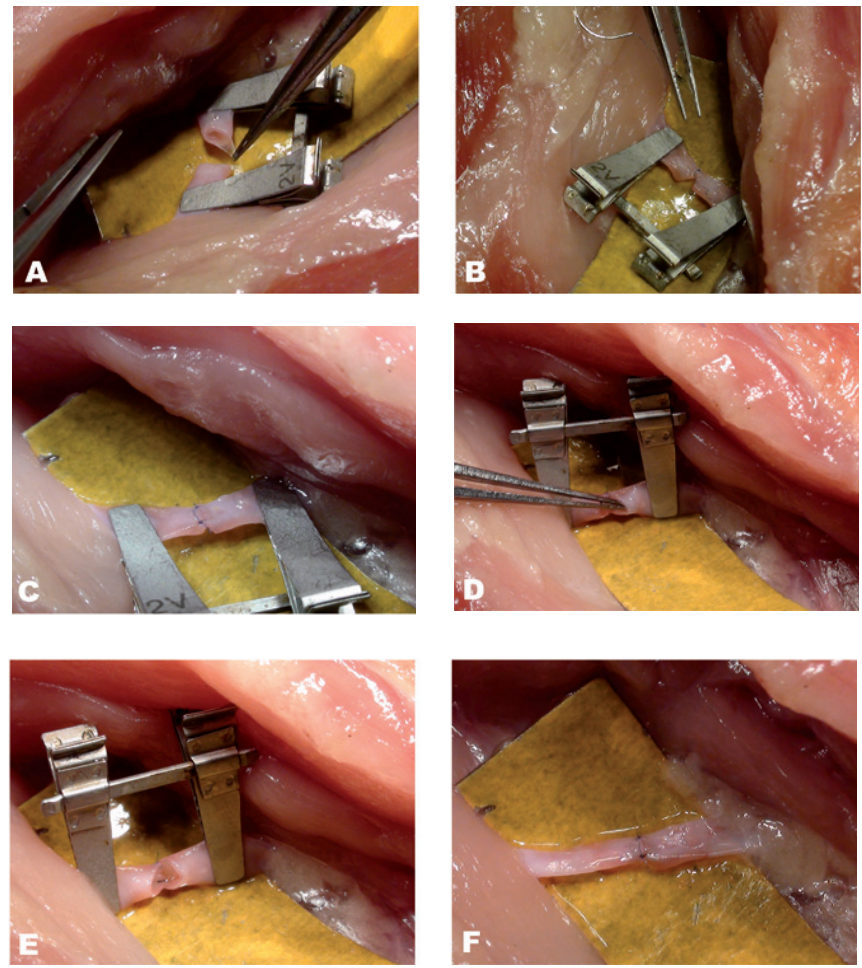

Figura 4 - Representação da etapa 3 a do protocolo. A: Ressecção da camada adventícia; $\boldsymbol{B}$ e $\boldsymbol{C}$ : Realização de suturas no vaso; D: Dilatação da luz do vaso; $\boldsymbol{E}$ : Rotação do clamp para a sutura na parede posterior do vaso; $\boldsymbol{F}$ : Suturas realizadas na circunferência do vaso.

e) Realize a dilatação da luz dos cotos vasculares;

f) Utilize o dilatador dentro da luz do vaso, como anteparo, para prosseguir com a sutura (Figura 4D);

g) Realize a sutura (Figura 4) de acordo com o item i da etapa $2 b$;

h) Retire o clamp distal e observe a patência da sutura;

i) Retire o clamp proximal.

\section{RESULTADOS}

As etapas do treinamento propostas se mostraram de fácil execução e baixo custo.

Os residentes do Serviço de Cirurgia Plástica da UFC que foram submetidos ao treinamento inicial o consideraram satisfatório, tendo contemplados todos os objetivos das fases propostas.

\section{DISCUSSÃO}

O desenvolvimento de habilidades microcirúrgicas é um processo lento, e contínuo, o qual requer imensa dedicação para o seu êxito. Pela complexidade do procedimento, o 
treinamento microcirúrgico sempre deve ser iniciado em um ambiente que não envolva pacientes, como, por exemplo, o laboratório ${ }^{14} \mathrm{e}$ o uso de luvas de látex é uma opção já bem estabelecida ${ }^{5,6,18}$. O uso deste material permite tanto o treinamento de suturas em incisões lineares quanto o de anastomoses, por meio da técnica de tubos de látex ${ }^{6}$.

Um obstáculo comum na organização de um centro de treinamento apropriado é o custo. Tanto os instrumentos quanto os equipamentos ópticos adequados ao treinamento são de custos elevados, o que desestimula tanto a instituição quanto o cirurgião principiante ${ }^{4,6,7}$. Nas etapas de anastomoses vasculares, surge ainda uma nova despesa, que é o de manutenção de um biotério e todos os demais custos inerentes à utilização de animais em pesquisas científicas ${ }^{6}$. Assim, a busca por novos modelos cuja barreira econômica seja menor e cujos resultados sejam igualmente satisfatórios deve ser sempre estimulada 6 .

Em um laboratório de microcirurgia, pode-se aprender a operar o microscópio, familiarizar-se com o campo bidimensional e com os instrumentos, e os princípios da técnica microcirúrgica. A prática experimental é indispensável na obtenção dos resultados desejados ${ }^{4,7}$.

$\mathrm{O}$ treinamento proposto exibe como vantagem evidente, a redução dos custos necessários à realização de um treinamento inicial. A exploração do látex como material fundamental no treinamento permite que este ultrapasse os limites de um laboratório, uma vez que pode ser realizado em qualquer ambiente, desde que se possua uma lupa binocular ${ }^{6}$. O uso de peças anatômicas provenientes de animais (Figuras 3 e 4) também contribui para a redução dos custos. Tais segmentos podem ser obtidos a partir de animais sacrificados para outros fins, como outras pesquisas em laboratórios ou adquiridos em locais em que essas peças sejam disponibilizadas para consumo.

Completar o treinamento básico não significa que o cirurgião estará apto a realizar procedimentos complexos no contexto clínico. É importante destacar que existem complicações que comprometem os resultados em microcirurgia e que somente com um treinamento continuado é possível minimizar estas ocorrências ${ }^{5}$.

\section{CONCLUSÃO}

O treinamento inicial proposto está em acordo com os requisitos expostos na literatura sobre o treinamento microcirúrgico. É indiscutível que a manutenção de um laboratório e de um biotério com fins experimentais representa uma significativa barreira econômica. A redução desta, que representa um obstáculo pertinente no Brasil, é especialmente importante, uma vez que facilita que um número maior de cirurgiões adquira conhecimentos práticos nesta técnica que ainda é tão carente de profissionais habilitados em nosso país.

\section{REFERÊNCIAS}

1. Yap LH, Butler CE. Principles of microsurgery. In: Beasley RW, Aston SJ, Bartlett SP, Gurtney GC, Spear SL, eds. Grabb and Smith's plastic surgery. Philadelphia: Lippincott Williams \& Wilkins;2007. p.66-72.

2. Ferreira MC, Marques E, Tedesco-Marchese AJ. Microcirurgia vascular: técnica para sutura de vasos com diâmetro externo inferior a $2 \mathrm{~mm}$. Rev Paul Med.1974;38(1):67-8.

3. Pessoa SGP, Riquet GF. Fundamentos básicos de microcirurgia vascular: estudo experimental. Ceará Médico.1982;4(1):10-6.

4. Webster R, Ely PB. Treinamento em microcirurgia vascular: é economicamente viável? Acta Cir Bras. 2002;17(3):194-7.

5. Martins PNA, Montero EFS. Basic microsurgery training. Comments and proposal. Acta Cir Bras. 2007; 22(1):79-81.

6. Pessoa BBGP, Pessoa SGP. Treinamento em microanastomoses utilizando tubos de látex. Acta Cir Bras. 2002;17(2):143-6.

7. Martins PNA, Montero EFS. Organization of a microsurgery laboratory. Acta Cir Bras. 2006;21(3):187-9.

8. Cunha MS, Ramos RS, Torres ALG, Souza DAM, Agra IMG, Eulálio JN. Aplicação da microcirurgia no Serviço de Cirurgia Plástica da Universidade Federal da Bahia: análise dos resultados e complicações. Rev Col Bras Cir. 2005;32(6):297-303.

9. Zhong T, Vaughan C, Bowen A. Microvascular surgical techniques. In: Malizos KN, editor. Reconstructive microsurgery. Texas: Landes Bioscience;2003. p.1-7.

10. Kallás IE, Kallás AC, Kallás E. Anastomoses arteriais: passado, presente e futuro. Acta Cir Bras. 1999;14(4):221-7.

11. O'Brien BM, Henderson PN, Bennett RC, Crock GW. Microvascular surgical technique. Med J Aust. 1970;1(14):722-5.

12. Figueiredo JCA, Pelosi MR. Princípios gerais de microcirurgia. In: Melega JM, eds. Cirurgia Plástica fundamentos e arte: princípios gerais. Rio de Janeiro:Medsi;2002. p.471-6.

13. Acland RD. Equipment for microsurgical practice. In: Acland RD, editor. Microsurgery practice manual. St. Louis: C.V. Mosby;1980. p.7-16.

14. Pessoa BBGP, Pessoa SGP. O retalho hipogástrico cutâneo no cão: modelo para o aprendizado experimental de microcirurgia. Acta Cir Bras. 2002;17(3):198-202.

15. Cunha MS, Torre ALG, Anjos Neto JC, Monteiro LL, Meneses JV. Transplantes microcirúrgicos: experiência de 5 anos do Serviço de Cirurgia Plástica da Universidade Federal da Bahia. Rev Bras Cir Plást. 2008;23(4):305-9.

16. Souza Filho MVP, Santos CC. Microcirurgia em reconstruções complexas: análise dos resultados e complicações. Rev Bras Cir Plást. 2009;24(2):123-30.

17. Torres ALG, Milcheski DA, Nakamoto HA, Tuma Jr. P, Ferreira MC. Aplicação da microcirurgia no reparo de lesões complexas. Rev Bras Cir Plást. 2009;24(2):131-7.

18. MacDonald JD. Learning to perform microvascular anastomosis. Skull Base. 2005;15(3):229-40.

\footnotetext{
Correspondência para:
} 